\title{
Brainstem hemorrhage is uncommon and is associated with high morbidity, mortality, and prolonged hospitalization
}

Renad M. AlMohammedi, MBBS, Hadeel AlMutairi, MBBS, Rana O. AlHoussien, MBBS, Malack T. AlOtayan, MBBS, Abeer K. AlMutairi, MBBS, Wejdan O. Bafail, MBBS, Altaf Khan, MS, Ismail A. Khatri, MD, FAAN.

\begin{abstract}

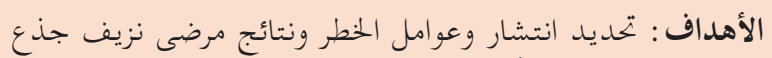

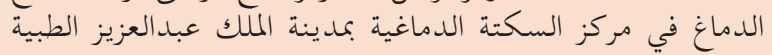
بالرياض

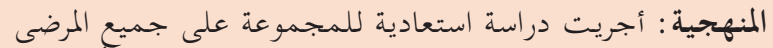

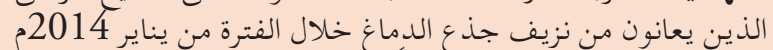

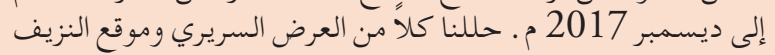

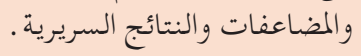

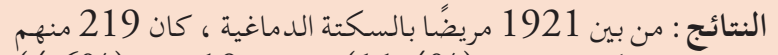

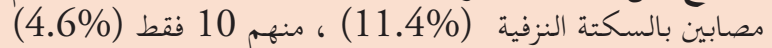

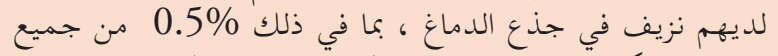

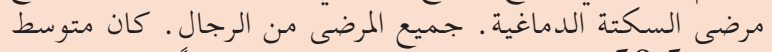

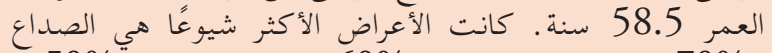

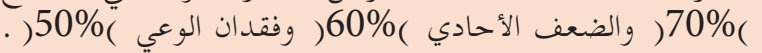

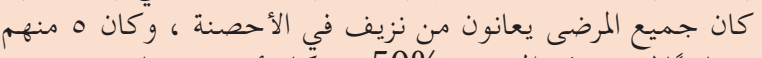

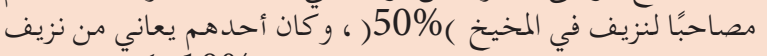

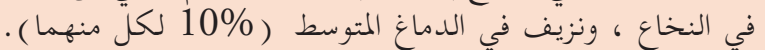

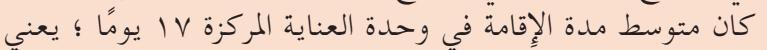

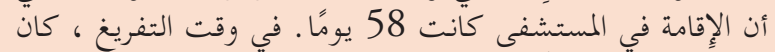

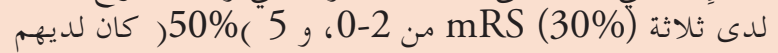
mRS

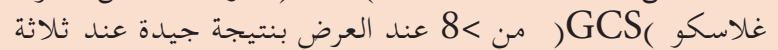

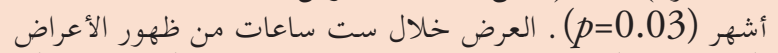
،

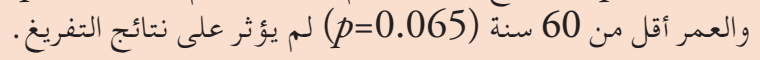

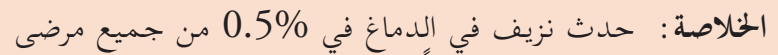

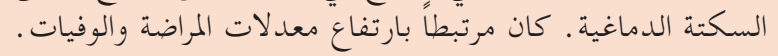

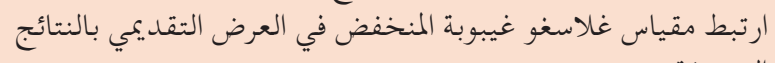
الضعيفة
\end{abstract}

Objectives: To determine the frequency, risk factors, and outcomes of patients with brainstem hemorrhage in stroke center at King Abdulaziz Medical City, Riyadh.
Methods: A retrospective, observational cohort study including all patients with brainstem hemorrhage from January 2014 to December 2017. The clinical presentation, location of hemorrhage, complications and clinical outcomes were analyzed.

Results: Of 1921 stroke patients, 219 had hemorrhagic stroke $(11.4 \%)$, of whom only 10 (4.6\%) had brainstem hemorrhage, comprising $0.5 \%$ of all stroke patients. All patients were men; mean age was 58.5 years. Most frequent presenting symptoms were headache $(70 \%)$, unilateral weakness $(60 \%)$, and loss of consciousness (50\%). All patients had hemorrhage in pons, 5 had concomitant cerebellar hemorrhage $(50 \%)$, one had medullary hemorrhage, and one midbrain hemorrhage (10\% each). Mean ICU stay was 17 days; mean hospital stay was 58 days. At the time of discharge, three $(30 \%)$ had $\mathrm{mRS}$ of $0-2,5(50 \%)$ had mRS of 3-5, whereas 2 (20\%) had died. Glasgow coma scale (GCS) of $>8$ at presentation was associated with a good outcome at three months $(p=0.03)$. Presentation within six hours of symptom onset $(p=0.233)$, hypertension on presentation $(p=0.233)$, and age less than 60 years $(p=0.065) \mathrm{did}$ not affect discharge outcomes.

Conclusion: Brainstem hemorrhage occurred in $0.5 \%$ of all stroke patients. It was associated with high morbidity and mortality. Low Glasgow Coma Scale at presentation was associated with poor outcomes.

Neurosciences 2020; Vol. 25 (2): $91-96$ doi: 10.17712/nsj.2020.2.20190102

From the Division of Neurology (AlMohammedi, Khatri), Department of Medicine, King Abdulaziz Medical City, College of Medicine (AlMutairi H, AlHoussien, AlOtayan, AlMutairi A, Bafail, Khatri), King Saud bin Abdulaziz University for Health Sciences, and from King Abdullah International Medical Research Center (Khan, Khatri), Riyadh, Kingdom of Saudi Arabia.

Received 23rd October 2019. Accepted 12th December 2019.

Address correspondence and reprint request to: Dr. Ismail A. Khatri, Division of Neurology, Department of Medicine, King Saud bin Abdulaziz University for Health Sciences, Riyadh, Kingdom of Saudi Arabia.E-mail: ismailkhatri@yahoo.com

ORCID ID: https://orcid.org/0000-0002-0218-18 
Stroke is among the leading causes of death and $\checkmark$ disability worldwide. In 2013, stroke was the second most common cause of death, accounting for $11.8 \%$ of all deaths worldwide, second only to ischemic heart disease. ${ }^{1}$ In Saudi Arabia, the reported prevalence of stroke is low compared to the Western countries, probably due to the predominance of a younger population in this region. One early study reported that the crude incidence of first-ever stroke in Saudi Arabia was 29.8/100,000/year, whereas the standardized incidence was $125.8 / 100,000 /$ year. $^{2}$ Stroke is pathologically classified into ischemic stroke, and hemorrhagic stroke. Ischemic stroke includes cerebral, retinal and spinal infarction, whereas hemorrhagic stroke typically includes intracerebral hemorrhage and subarachnoid hemorrhage. ${ }^{3}$ Intracerebral hemorrhage frequency ranges from $6.5 \%$ to $19.6 \%$ of all strokes and is associated with high mortality. ${ }^{4,5}$ Intracerebral hemorrhage $(\mathrm{ICH})$ is mostly caused by the rupture of arteries due to hypertensive changes or other vascular abnormalities. ${ }^{6}$

The incidence of hypertensive ICH has decreased in the developed countries with better blood pressure control, whereas the burden of ICH has increased in the developing countries. ${ }^{4,7}$ In an analysis of 500 consecutive cases from a hospital-based study in Saudi Arabia, $\mathrm{ICH}$ was detected in $21.4 \% .{ }^{8}$ Brainstem hemorrhage is an uncommon form of ICH. In one study, 60 of 1033 patients with $\mathrm{ICH}$ were diagnosed with pontine hemorrhage - an incidence of $5.8 \%$. $^{9}$

The outcome for $\mathrm{ICH}$ is variable, depending on the age, use of anticoagulants, volume or expansion of hematoma, and extension to ventricles. ${ }^{10} \mathrm{ICH}$ has higher mortality and disability than ischemic stroke. ${ }^{11}$ Only $12 \%$ to $39 \%$ of patients with $\mathrm{ICH}$ have a good prognosis. ${ }^{11}$ In a meta-analysis of 122 eligible studies, it was found that less than a third of patients with $\mathrm{ICH}$ survive 5 years and less than half of them survive one year. ${ }^{12}$ In Saudi Arabia, 31\% of stroke patients died as an eventual outcome. ${ }^{13}$ Moreover, in this Saudi study, stroke patients had an average hospital stay of 63 days. ${ }^{13}$

There is a lack of Saudi Arabian studies about the frequency and outcome of stroke. ${ }^{14}$ These kinds of studies have a great impact on understanding the disease and improving the health care practice and awareness. ${ }^{14}$

Disclosure. Authors have no conflict of interests, and the work was not supported or funded by any drug company.
We found some local studies that studied frequency and subtypes of stroke including brainstem hemorrhage, but were not specific to brainstem hemorrhage. ${ }^{8,13}$ The pattern of each subtype was not clear in the mentioned studies. They lack the specificity of studying brainstem hemorrhage, its clinical presentation, the outcomes, and the complications. So, this study aimed to determine the frequency and the risk factors of brainstem hemorrhage among all adult stroke patients. It also investigated the in-hospital complications and the clinical outcomes at discharge and at 3 months.

Methods. The study was approved by the Institutional Review Board (IRB) of King Abdullah International Medical Research Center (KAIMRC). It was conducted in the stroke unit of the King Abdulaziz Medical City (KAMC), Ministry of National Guard Health Affairs (MNGHA), in Riyadh, Saudi Arabia. It was a retrospective, observational cohort study from January 2014 to December 2017. The IRB waived the need for consent from patients due to retrospective chart review nature of the study. Brainstem hemorrhage, hemorrhagic stroke, intracerebral hemorrhage, and Saudi Arabia were used as key terms to search local studies about brainstem hemorrhage from various databases including PubMed, google scholar, Research Gate, as well the Saudi Ministry of Health Open Data. ${ }^{15}$

Inclusion/exclusion criteria. All patients above the age of 16 years, of both genders, with a final diagnosis of brainstem hemorrhage were included. Patients with ischemic stroke and with hemorrhagic stroke primarily outside the brainstem were excluded. Patients with other types of brain hemorrhage, for example, subdural hemorrhage and subarachnoid hemorrhage, were also excluded. Brainstem hemorrhage was defined as any hemorrhage where the epicenter of the hemorrhage was considered to be in the brainstem (midbrain, pons, medulla) or in the cerebellum with involvement of any part of the brainstem. Patients with intracerebral hemorrhage but without involvement of the brainstem were excluded. Patients with intracerebral hemorrhage in whom the involvement of the brainstem was an extension of the ICH from other locations were also excluded. All brainstem hemorrhage etiologies were included except where subarachnoid hemorrhage was the primary mechanism. Intensive care unit (ICU) and hospital stays were recorded, and in-hospital complications were identified. Outcome was determined at discharge and at three months using the modified Rankin Scale (mRS) score. The mRS was determined by neurologists or residents and fellows in neurology 
Table 1 - Frequency of presenting symptoms among patients with hemorrhagic stroke. $\mathrm{N}=10$

\begin{tabular}{ll}
\hline Symptoms & $\mathbf{n}(\%)$ \\
\hline Unilateral weakness & $6(60)$ \\
Unilateral numbness & $2(20)$ \\
Loss of speech & $3(30)$ \\
Loss of consciousness & $5(50)$ \\
Altered level of consciousness & $2(20)$ \\
Sudden vertigo & $1(10)$ \\
Sudden balance problems & $1(10)$ \\
Sudden severe headache & $7(70)$ \\
Seizure/fit & $2(20)$ \\
Vomiting & $2(20)$ \\
\hline
\end{tabular}

Table 2 - Frequency of stroke related complications. N=10

\begin{tabular}{ll}
\hline Complications & n (\%) \\
\hline Seizures & $2(20)$ \\
Pneumonia & $2(20)$ \\
Urinary tract infections & $4(40)$ \\
Sepsis & $2(20)$ \\
Bedsores & $1(10)$ \\
Gastrostomy tube placement & $2(20)$ \\
Tracheostomy tube placement & $3(30)$ \\
Limb contractures & $1(10)$ \\
Death & $2(20)$ \\
\hline
\end{tabular}

training during direct patient contact.

The data were analyzed using SAS version 9.4. Descriptive statistics were presented as frequencies and percentages for categorical data. Numerical data were presented by means and standard deviations. The Pearson chi-square test was utilized to analyze the associations. $P$-value less than or equal to 0.05 was considered statistically significant.

Results. A total of 1921 patients were admitted to the stroke unit during the period January 2014 to December 2017; of these, 219 patients had hemorrhagic stroke (11.4\%). Among the patients with hemorrhagic stroke, only 10 patients had brainstem hemorrhage $(4.6 \%$ of all hemorrhagic strokes, and $0.5 \%$ of all strokes). All patients with brainstem hemorrhage were men, the mean age was $58.5 \pm 13.1$ years and the mean BMI was $27.8 \pm 6.2 \mathrm{~kg} / \mathrm{m}^{2}$. Half the patients were younger than 60 years. Two patients were brought to the hospital by ambulance, 6 patients by private transport, and 2 patients were transferred from another hospital.

Six of the 10 patients presented within 6 hours of symptom onset, whereas 2 presented more than 24 hours after symptom onset. The most frequent presenting symptoms are shown in Table 1 . Common vascular risk factors included hypertension in $90 \%$, diabetes in $80 \%$, congestive heart failure in $30 \%$, ischemic heart disease in $20 \%$, prior stroke and smoking in 30\% each, and dyslipidemia in $10 \%$. One patient $(10 \%)$ was on anticoagulation medication and one patient (10\%) had valvular heart disease. Five (50\%) patients had GCS below 8 at the time of presentation. Seven (70\%) patients had focal weakness, one (10\%) had focal numbness, 3 (30\%) had absent brainstem reflexes, and $5(50 \%)$ patients had pupillary abnormality. More than half $(60 \%)$ of the patients presented with BP $>160 / 100$ $\mathrm{mm} \mathrm{Hg}$. Nine patients had a high blood glucose reading $(90 \%)$, and the $\mathrm{HbA1c}$ ranged between $6.1 \%$ and $11.6 \%$, with a mean of $6.4 \%$.

Hemorrhagic stroke related complications are shown in Table 2. When evaluating the anatomical distribution of the brainstem hemorrhage, all the 10 patients had hemorrhage in pons, $5(50 \%)$ had concomitant cerebellar hemorrhage, one (10\%) had medullary hemorrhage, and one (10\%) had midbrain hemorrhage. Three (30\%) had intraventricular extension of the hemorrhage.

The mean ICU stay of patients with brainstem hemorrhage was $17 \pm 34$ days; while the mean length of hospital stay was $58 \pm 71$ days. Half of the patients $(50 \%)$ were discharged home, one patient $(10 \%)$ was discharged to an inpatient rehabilitation center, one patient $(10 \%)$ was discharged to a long-term facility/ nursing home, and one patient (10\%) was still admitted at 3 months. There was no statistically significant difference in the discharge disposition between the patients below 60 years of age and those above 60 years of age ( $p=0.06)$ The relationship of age, durations of symptoms and presentation Glasgow Coma Scale (GCS) to discharge disposition is shown in Table 3.

Discussion. Brainstem hemorrhage is an uncommon type of hemorrhagic stroke. Our study looked at the frequency and clinical outcomes of brainstem hemorrhage in a local population. Among all stroke patients, only $0.5 \%$ had brainstem hemorrhage. Among the patients with hemorrhagic stroke, only $4.6 \%$ had brainstem hemorrhage. This frequency, although very low, was not totally unexpected as other studies have shown a similar low prevalence of brainstem hemorrhage. ${ }^{9,16}$

Pontine hemorrhage was the most common brainstem hemorrhage, found in all ten patients. Only $10 \%$ of the patients had concomitant midbrain hemorrhage, $10 \%$ had medullary hemorrhage, $50 \%$ 
Table 3 - Comparative analysis according to the age, duration of symptoms, and Glasgow Coma Scale.

\begin{tabular}{|c|c|c|c|c|c|c|c|c|c|c|}
\hline \multirow{2}{*}{$\begin{array}{l}\text { Outcomes } \\
\text { Discharge disposition }\end{array}$} & \multicolumn{3}{|c|}{ Age } & \multicolumn{3}{|c|}{ Duration of symptoms } & \multicolumn{4}{|c|}{ Glasgow Coma Scale } \\
\hline & $<60$ years & $>60$ years & $P$-value & $<6$ hours & $>6$ hours & $P$-value & $<8$ & $8-12$ & $13-15$ & $P$-value \\
\hline To home & $20 \%$ & $80 \%$ & 0.06 & $50 \%$ & $50 \%$ & 0.15 & $20 \%$ & $75 \%$ & $100 \%$ & 0.31 \\
\hline To inpatient rehabilitation & $20 \%$ & 0 & & 0 & $25 \%$ & & 0 & $25 \%$ & 0 & \\
\hline $\begin{array}{l}\text { To long term facility/nursing } \\
\text { home }\end{array}$ & 0 & $20 \%$ & & $16.7 \%$ & 0 & & $20 \%$ & 0 & 0 & \\
\hline Admitted at 3 months & $20 \%$ & 0 & & 0 & $25 \%$ & & $20 \%$ & 0 & 0 & \\
\hline Dead & $40 \%$ & 0 & & $33.3 \%$ & 0 & & $40 \%$ & 0 & 0 & \\
\hline \multicolumn{11}{|c|}{ Modified Rankin scale at discharge } \\
\hline $0-2$ & $20 \%$ & $40 \%$ & 0.19 & $16.7 \%$ & $50 \%$ & 0.23 & 0 & $75 \%$ & 0 & 0.05 \\
\hline $3-5$ & $40 \%$ & $60 \%$ & & $50 \%$ & $50 \%$ & & $60 \%$ & $25 \%$ & $100 \%$ & \\
\hline 6 (death) & $40 \%$ & 0 & & $33.3 \%$ & 0 & & $40 \%$ & 0 & 0 & \\
\hline \multicolumn{11}{|c|}{ Modified Rankin scale at 3 months } \\
\hline $0-2$ & 0 & $20 \%$ & 0.13 & 0 & $25 \%$ & 0.16 & 0 & $25 \%$ & 0 & 0.03 \\
\hline $3-5$ & $20 \%$ & $60 \%$ & & $50 \%$ & $25 \%$ & & $60 \%$ & 0 & $100 \%$ & \\
\hline 6 (death) & $40 \%$ & 0 & & $33.3 \%$ & 0 & & $40 \%$ & 0 & 0 & \\
\hline No follow up & $40 \%$ & $20 \%$ & & $16.7 \%$ & $50 \%$ & & 0 & $75 \%$ & 0 & \\
\hline
\end{tabular}

had cerebellar hemorrhage, and 30\% had ventricular extension. These findings were consistent with prior observations of a higher frequency of pontine and cerebellar hemorrhage relative to other parts of the brainstem. ${ }^{17}$ A study looking at cerebral microbleeds and $\mathrm{ICH}$ among 79 patients with vertebrobasilar dolichoectasia found that only two of the patients had microbleeds in the medulla oblongata and 6 of them had microbleeds in the pons. ${ }^{17}$

All brainstem hemorrhage patients in our cohort were men, with a mean age of $58.5 \pm 13.1$ years. Male gender and older age are considered risk factors for hemorrhagic stroke, including brainstem hemorrhage. Other studies, including a systemic review, have found male gender to be a risk factor for hemorrhagic stroke. ${ }^{1,16,18}$ In a study conducted in Japan, it was shown that patients with pontine hemorrhage were older in age. ${ }^{16}$ This study ${ }^{16}$ also found that older age was related to poor outcomes and high mortality, while our results showed no significant relation between age and outcomes. We suggest that this difference was possibly due to the small size of our cohort.

The mean BMI of our patients was $27.8 \pm 6.2 \mathrm{~kg} / \mathrm{m}^{2}$, which categorizes most of our patients as obese. This may suggest a correlation between higher BMI and brainstem hemorrhage. A prior study looking at the relationship between BMI and strokes in general, suggests that high $\mathrm{BMI}$ is a risk factor for both hemorrhagic and ischemic strokes. ${ }^{19}$

Among the vascular risk factors, hypertension was found in $90 \%$ of our patients, which is a typical risk factor for hemorrhagic stroke; however, most studies of hemorrhagic stroke report a much smaller frequency of hypertension. ${ }^{18}$ This high number may be due to the very small sample. In our cohort, $30 \%$ of the patients had previous strokes. Diabetes mellitus was found in 8 out of our 10 patients, which accounted for a large majority $(80 \%)$ as compared to the usual frequency of about 30\% in stroke patients. This again may be related to the small sample with high vascular comorbidities.

At the time of presentation, $60 \%$ of the patients had blood pressure above 160/100 $\mathrm{mm} \mathrm{Hg}, 50 \%$ had a GCS score $<8$, and $70 \%$ presented with a focal weakness. High blood pressure, low GCS score, and focal weakness have all been reported to be common at the time of presentation of hemorrhagic stroke. ${ }^{20} \mathrm{We}$ found that low GCS scores were significantly related to high morbidity at the time of discharge and at 3 months. This association was consistent with a study conducted in Japan that showed that low GCS is a predictive factor for poor outcomes in patients with pontine hemorrhage. ${ }^{16}$ 
In our cohort, all the patients developed one or more stroke related complications including seizures, hospital acquired pneumonia, urinary tract infection, sepsis, tracheostomy tube placement, and two patients died. In addition to these complications, the length of both the ICU stay and the hospital stay were prolonged for all patients. The mean length of ICU stay was 16.8 days, while the mean length of hospital stay was 58 days. According to Chan et $\mathrm{al}^{21}{ }^{21}$ the definition of a prolonged ICU length of stay is equal to or more than 10 days, which indicates that nearly half of our patients (40\%) had a prolonged ICU length of stay. The patients with brainstem hemorrhage had significant disability and were not considered candidates for inpatient rehabilitation, whereas families were reluctant to take these patients home due to limited support services available at home to take care of these dependent patients. This may have contributed significantly to the prolonged hospitalization in our patients.

Our study showed that brainstem hemorrhage had very low frequency among all strokes, as well as among hemorrhagic strokes, and was associated with many complications and morbidity. It also showed that a low GCS score at time of presentation was associated with a poor outcome. Some of the strengths of our study included its large cohort, of almost 2000 stroke patients over 4 years, from January 2014 to December 2017. We looked at presentation, anatomical location, in-hospital complications, and outcomes. Follow-up at 3 months was available for most patients.

One of the major limitations of our study was its retrospective nature as this limited the interpretation of causality. Due to the small number of patients with brainstem hemorrhage, a number of associations could not be tested statistically. Incomplete documentation in charts also limited the data collection to some extent.

Conclusions and recommendations. Our study showed that brainstem hemorrhage was a very infrequent type of intracerebral hemorrhage. It was found in only $0.5 \%$ of stroke patients. It was associated with poor outcomes and high complication rates. Low GCS at presentation was a determinant of poor outcomes. Men were exclusively affected in our study, while age did not make a difference to outcome. The majority of our patients had a long intensive care unit stay and a mean length of hospital stay. Our findings add to the knowledge and pool of information about brainstem hemorrhage. Our study confirms the rarity and the poor prognosis, and confirms the association between low GCS and the outcome. Longer duration of study, or multicenter studies, could increase the cohort size for this extremely uncommon but potentially lifethreatening and disabling type of hemorrhagic stroke and help understand brainstem hemorrhage better in Saudi Arabia.

Acknowledgment. We would like to thank Dr. Misealreem Shaheen, College of Medicine, King Saud bin Abdulaziz University for Health Sciences for assistance with data analysis. We would like to thank Scribendi Inc. Chatham, Ontario N7M ON3, Canada for English language editing.

\section{References}

1. Feigin VL, Norrving B, Mensah GA. Global burden of stroke. Circ Res 2017; 120: 439-448.

2. al-Rajeh S, Larbi EB, Bademosi O, Awada A, Yousef A, al-Freihi $\mathrm{H}$, et al. Stroke register: experience from the eastern province of Saudi Arabia. Cerebrovasc Dis 1998; 8: 86-89.

3. Hankey GJ. Stroke. Lancet 2017; 389: 641-654.

4. Feigin VL, Lawes CM, Bennett DA, Barker-Collo SL, Parag $\mathrm{V}$. Worldwide stroke incidence and early case fatality reported in 56 population-based studies: a systematic review. Lancet Neurol 2009; 8: 355-369.

5. Sacco S, Marini C, Toni D, Olivieri L, Carolei A. Incidence and 10 -year survival of intracerebral hemorrhage in a populationbased registry. Stroke 2009; 40: 394-399.

6. Veltkamp R, Purrucker J. Management of Spontaneous Intracerebral Hemorrhage. Curr Neurol Neurosci Rep 2017; 17: 80 .

7. Hong KS, Bang OY, Kang DW, Yu KH, Bae HJ, Lee JS, et al. Stroke statistics in Korea: part I. Epidemiology and risk factors: a report from the korean stroke society and clinical research center for stroke. J Stroke 2013; 15: 2-20.

8. al Rajeh S, Awada A, Niazi G, Larbi E. Stroke in a Saudi Arabian National Guard community. Analysis of 500 consecutive cases from a population-based hospital. Stroke 1993; 24: 16359.

9. Nakajima K. Clinicopathological study of pontine hemorrhage. Stroke 1983; 14: 485-493.

10. Hemphill JC 3rd, Bonovich DC, Besmertis L, Manley GT, Johnston SC. The ICH score: a simple, reliable grading scale for intracerebral hemorrhage. Stroke 2001; 32: 891-897.

11. An SJ, Kim TJ, Yoon BW. Epidemiology, risk factors, and clinical features of intracerebral hemorrhage: an update. $J$ Stroke 2017; 19: 3-10.

12. Poon MT, Fonville AF, Al-Shahi Salman R. Long-term prognosis after intracerebral haemorrhage: systematic review and meta-analysis. J Neurol Neurosurg Psychiatry 2014; 85: 660-667.

13. Qari FA. Profile of stroke in a teaching university hospital in the western region. Saudi Med J 2000; 21: 1030-1033.

14. Robert AA, Zamzami MM. Stroke in Saudi Arabia: a review of the recent literature. Pan Afr Med J 2014; 17: 14.

15. Ministry of Health - Saudi Open Data [Internet]. Data.gov.sa. 2020 [cited 15 November 2019]. Available from: https://data. gov.sa/Data/en/organization/ministry_of_health

16. Nishizaki T, Ikeda N, Nakano S, Sakakura T, Abiko M, Okamura T. Factors determining the outcome of pontine hemorrhage in the absence of surgical intervention. Open Journal of Modern Neurosurgery 2012; 2: 17-20. 
17. Förster A, Wenz R, Maros ME, Böhme J, Al-Zghloul M, Alonso A, et al. Anatomical distribution of cerebral microbleeds and intracerebral hemorrhage in vertebrobasilar dolichoectasia. PLoS One 2018; 13: e0196149.

18. Ariesen MJ, Claus SP, Rinkel GJ, Algra A. Risk factors for intracerebral hemorrhage in the general population: a systematic review. Stroke 2003; 34: 2060-2065.

19. Song YM, Sung J, Davey Smith G, Ebrahim S. Body mass index and ischemic and hemorrhagic stroke: a prospective study in Korean men. Stroke 2004; 35: 831-836.
20. Ojaghihaghighi S, Vahdati SS, Mikaeilpour A, Ramouz A. Comparison of neurological clinical manifestation in patients with hemorrhagic and ischemic stroke. World J Emerg Med 2017; 8: 34-38.

21. Chan CL, Ting HW, Huang HT. The definition of a prolonged intensive care unit stay for spontaneous intracerebral hemorrhage patients: an application with national health insurance research database. BioMed Res Int. 2014; 2014: 891725.

\section{Authorship entitlement}

Excerpts from the Uniform Requirements for Manuscripts Submitted to Biomedical Journals updated November 2003.

Available from www.icmje.org

The international Committee of Medical Journal Editors has recommended the following criteria for authorship; these criteria are still appropriate for those journals that distinguish authors from other contributors.

Authorship credit should be based on 1) substantial contributions to conception and design, or acquisition of data, or analysis and interpretation of data; 2) intellectual content; and 3) final approval of the version to be published. Authors should meet conditions 1,2 , and 3 .

Acquisition of funding, collection of data, or general supervision of the research group, alone, does not justify authorship.

An author should be prepared to explain the order in which authors are listed. 